\title{
How the Development, Features and Roll-Out of a SARS-COV-2 Vaccine Shape Public Acceptance: A Conjoint Experiment in a Large Representative Sample of Danes
}

Frederik Juhl Jørgensen ( $\square$ fj@ps.au.dk)

Aarhus University

Alexander Bor

Aarhus University

Michael Bang Petersen

Aarhus University

Research Article

Keywords: Vaccine acceptance, vaccine hesitancy, conjoint experiment

Posted Date: February 24th, 2022

DOI: https://doi.org/10.21203/rs.3.rs-1237898/v1

License: (1) This work is licensed under a Creative Commons Attribution 4.0 International License. Read Full License 


\section{Abstract \\ Background}

While effective vaccines against the SARS-COV-2 virus have been developed and countries around the world have invested heavily to secure vaccine rollout, a fundamental challenge remains. How do policy-makers around the world ensure high vaccine uptake? What is lacking is a comprehensive assessment that captures a total spectrum of features related to the development of a vaccine, the vaccine's characteristics as well as the implementation of the vaccination program.

\section{Methods}

To provide such an assessment, we designed a conjoint experiment embedded in large-scale surveys based on a random sample from the central database of Danish social security numbers $(\mathrm{N}=3,099)$, providing a sample that is representative for the adult Danish population. In the conjoint experiment, we vary features relating to three dimensions: 1 ) the stage of vaccine development, 2) the specific characteristics of the vaccine, and 3) the implementation of the vaccination strategy.

\section{Results}

We show that the features relating to characteristics of the specific vaccine have the strongest impact on vaccine acceptance. The features relating to vaccine development were the second most powerful, while the features relating to the implementation of the vaccination strategy were the least.

\section{Conclusions}

The public has a strong preference for vaccines with high efficacy. This may point to a mounting challenge for ensuring high vaccine uptake. Yet, the results also provide evidence that there might be a pathway to naturally alleviate this challenge. Thus, over time the "testing period" of the vaccines are naturally extended, which simultaneously implies an increase in vaccine safety (provided that the roll-out only reveals marginal side-effects). Our results suggest that such an increase in vaccine safety in itself will increase acceptance (in particular, if the roll-out only reveals marginal side-effects). Furthermore, the results indicate that vaccine acceptance can be increased if politicians leave it to the health authorities to communicate about recommendations.

\section{Full Text}

Vaccines are vital tools in the fight against the SARS-COV-2 virus [1]. Accordingly, pharmaceutical companies have worked at hitherto unseen speed and invested unprecedented amounts of resources into the successful development of effective vaccines against the virus. Similarly, we have seen that countries around the world are investing heavily in the necessary infrastructure to secure the rollout and distribution of these vaccines to their populations. However, the development of effective vaccines and the necessary channels for their distribution does not necessarily mean an end to the pandemic. A fundamental challenge, for policymakers around the world, is hence to ensure high vaccine uptake. Thus, immunization programs will not have the desired effects if large proportions of populations around the world are not willing to take the vaccines they are offered [2]. This challenge is emphasized by numerous studies that point out that significant proportions of the public are hesitant about SARS-COV-2 vaccines $[3,4,5,6]$. Moreover, the emergence of more contagious and potentially vaccine-resistant SARS-COV-2 virus variants might require that significant proportions of the public need to be re-vaccinated with second-generation vaccines to reach herd-immunity [2].

Previous work in the context of SARS-COV-2 has found that vaccine acceptance differ substantially across individuals based on their specific demographic and psychological traits. Thus, studies have found that males $[5,7,8,9]$, older people $[4,5,9]$, and the better educated $[5,9]$ are more likely to accept a SARS-COV-2 vaccine. Moreover, studies have also found that trust in authorities $[5,6]$ and trust in others $[5,10]$ are key predictors of SARS-COV-2 vaccine acceptance. Studies focusing on risk-perceptions, find that both personal risk-perceptions $[5,7,8,9]$ as well as sociotropic risk-perceptions $[5,11]$ are key predictors of vaccine 
acceptance such that those who feel more at risk are more likely to accept vaccines. Finally, prior literature has linked attitudinal predictors relating to anti-systemic sentiments $[12,13]$ and feelings of pandemic fatigue $[5,14]$ to SARS-COV-2 vaccine acceptance.

While the role of individual traits is highly important for understanding vaccine acceptance, these traits are also relatively immutable. Health communicators and authorities motivated to increase vaccine acceptance in the general public accordingly need to invest elsewhere to meet their goals, at least in the short term. In this respect, the features of the vaccines themselves are key. Several theoretical frameworks for understanding vaccine acceptance converge on the argument that (a) the development and approval process of a specific vaccine needs to inspire confidence; (b) the characteristics of the vaccine in terms of effectiveness and safety should remove complacency and, hence, make it a worthwhile calculation to receive vaccination; and (c) that the actual implementation of the vaccination program should increase convenience and, thus, reduce potential practical constraints that disables an individual from acting on their intention to get vaccinated $[15,16]$.

Consistent with this, four rapidly executed studies on the question of vaccine characteristics has utilized conjoint experiments to assess some of these three dimensions (confidence, complacency and convenience) and how their influence acceptance of a SARS-COV-2 vaccine. Consistent with previous studies on vaccine uptake (see e.g., reference [17] and references therein), these studies show that higher efficacy leads to higher uptake [18, 19,20,21]. Moreover, similar to prior research on other vaccines [22, $23,24,25]$, three of the studies show that an increased risk of side effects decrease vaccine uptake [19,20,21], while one study do not find this effect [18]. Consistent with prior research, the process of development has also been found to affect uptake [26]. While reference [18] do not investigate this effect, the other three studies consistently find an impact of production country on uptake [19, 20, 21]. Relatedly, reference [21] also examine the impact of an increased development period and finds small positive effects on uptake.

While these findings provide important first steps in understanding how features of vaccines and vaccinations programs themselves work beyond individual traits to increase acceptance, the studies also face noteworthy limitations. First, three of the studies $[18,20,21]$ focus only on of the vaccines themselves, leaving the question of the implementation of the vaccination strategy unanswered. Second, these three studies are all focused only on the USA. This questions whether their estimates generalize to other contexts with, for example, less politicization of COVID-19. Finally, the three studies are based on convenience samples, which further questions the generalizability of the results beyond the samples. Reference [19] remedies some of these limitations. Focusing on the first weakness, the authors study a broader set of features, including also the place of vaccine administration and whether or not the GP recommends the vaccination. While this broadened focus is a strength compared to the other studies, there are additional factors in the national vaccination strategy that should be considered from a convenience perspective (as developed below). Moving to the two latter weaknesses, reference [19] move their study outside the American context and instead focus on France. Moreover, they were able to conduct their survey on a sample of 2000 adults aged 18-64 in France based on a probability sample that ensures representativeness for the working-age French population. Although their sampling overall should be viewed as a strength, it also has its limitations. Thus, they remain unable to observe effects among the most vulnerable groups-i.e., those who are $65+$ years old.

In this manuscript, we seek to resolve these limitations. What we lack is a more comprehensive assessment that captures a total spectrum of features related to the development of a vaccine, the vaccine's characteristics as well as the implementation of the vaccination program; hence, assessing the relative importance of both confidence, complacency and convenience as highlighted in frameworks of vaccine acceptance. To provide such an assessment, we designed a conjoint experiment embedded in highquality large-scale surveys focused on the Danish population. Note that these surveys were designed before the publication of all the studies above. Specifically, we collected a random sample of 3,099 Danes between November 27 and December 17 (i.e., prior to the start of the Danish vaccination program) based on the database of Danish social security numbers (providing a sample that is representative for the entire adult Danish population). In these surveys, we varied three features related to the stage of development of the vaccine: the number of previously vaccinated, length of testing period before approval, and production country. We varied two features related to the specific characteristics of the vaccine: its efficacy and expected side-effects. And we also manipulated four characteristics related to the implementation of the vaccination strategy: place of vaccination, vaccine recommendation, when you can be vaccinated, and whether you need an appointment or not. 
Our aim is first to provide a comprehensive assessment of which particular types of vaccines the public is willing to accept and whether certain vaccination strategy characteristics potentially foster acceptance. Second, we also aim to test whether these vaccine characteristics potentially diminish the influence of recognized individual-level predictors of vaccine acceptance. As accounted for above, we for example know that those who are personally worried are more likely to take a vaccination offer. A key concern thus might be that characteristics of the vaccines and vaccination strategy primarily affect those who are likely to already accept vaccines. This suggest interactions, where the effects of the vaccine and vaccination strategy attributes would be strongest for those who would already be most inclined to accept vaccination offers given their background characteristics. In the previous studies, this question remains understudied.

\section{Methods}

\section{Data}

We conducted a conjoint experiment among the Danish public aged 18 or older. Our data is collected by the survey firm Epinion. Participants were recruited using simple random sampling based on the entire database of Danish social security numbers, supplied by Statistics Denmark (DST). The recruited participants can thus be viewed as representative of the broader Danish population. We interviewed 3,099 individuals over two rounds. The first round of data was collected between November 27 and December 4, 2020 and included 1,547 individuals. The second round of data was collected between December 11 and December 17 and included 1,552 individuals. For each round, 4,000 Danes were randomly selected from the database of social security numbers.

Table S.1 in the supplementary material (SM) gives an overview of the data collection process. The randomly selected individuals received a letter inviting them to participate in the study via eBoks (a nation-wide Danish electronic mail system). If they had not taken the survey after two days, they received a letter reminding them about the study also via eBoks. If invitees did not respond upon this first reminder, they were contacted by text message or via the telephone and reminded about the study. About . 8 percent of the Danish population are exempt from eBoks. Individuals who were not signed up for eBoks (i.e., had an exemption), were instead contacted over the telephone and offered to do the interview. Overall, the average response rate in the study is 39 percent. After the data collection, we correct for small imbalances using post-stratification weights to adjust the final sample data to fit the demographic margins of the Danish population with respect to sex, age, education, and geography (see Table S.2 in the SM for detailed sample and population characteristics). We employ these post-stratification weights in all results in the manuscript, but note that results are essentially identical without this reweighting (see Figure S.4 in the SM).

\section{Research design}

We conduct a conjoint experiment among 3,099 individuals who assess four vaccine profiles each, yielding 12,396 observations in total. Conjoint experiments ask participants to evaluate (hypothetical) profiles that vary randomly on multiple attributes at the same time. Conjoints have been widely applied in marketing and, increasingly so, in other fields within social science to measure preferences and the relative importance of several determinants in multidimensional decision-making processes $[27,28,29,30]$. Similarly, conjoint experiments have been employed to study vaccine acceptance in previous epidemics and under the current pandemic $[18,19,20,21]$.

Just as in real-world vaccine decisions, in our experiment, participants are presented with a vaccine and then asked to decide whether they would take the vaccination or not. The vaccinations vary on nine attributes, including efficacy, side-effects, number already vaccinated, length of the testing period, production country, by whom it is recommended, vaccination place, when you can get vaccinated, and how you schedule an appointment. Each attribute can take on various values that are randomly chosen to form the vaccination. Table S.3 gives an overview of attributes and their values. The attributes were presented in a random order.

In addition to varying the profile of a vaccine, we also varied the context in which a vaccination was offered. Specifically, we varied whether the corona epidemic would increase, continue as now, or decrease. Participants saw a preamble reading: "Imagine that the corona epidemic in Denmark [increases/continues as now/decreases] and that you within the next month get an offer of this free vaccine against COVID-19, which is recommended for people like you." We randomly varied the context across participants, but not across profiles evaluations. In other words, a participant evaluated four randomly varying vaccines within the 
same context. Finally, participant were asked to evaluate whether or not they would take the vaccine asking the question: "Would you take the vaccine or would you reject the offer?" Figure S.1 in the SM presents an example of a random profile with the preamble and the outcome evaluation question.

As our benchmark model, we regress the probability of vaccine acceptance on the nine attributes in the same model pooling across all respondents and account for the fact that individuals evaluate several profiles by using standard errors clustered on individuals (see Fig. 1). Given randomization, this strategy provides unbiased estimates for the independent causal impact of each of the attributes because it ensures that the potential vaccine acceptance outcomes are independent of attribute assignment. An implication of randomization is that individual-level characteristics are expected to be balanced across attributes. One way of assessing the design-based identification is thus to include individual-level covariates into the model and evaluate whether the estimated effects change upon inclusion (Figure S.4 shows that the estimated effects remain unchanged upon inclusion of covariates, corroborating the design-based identification).

\section{Results}

\section{Epidemic context effects}

Across participants, we varied the context (i.e., the epidemic development) in which individuals decided whether to accept a given vaccine or not. In Figure S.2, we utilize this to test whether the epidemic context matters by regressing vaccine acceptance on the context variable. The figure shows only marginal differences in acceptance and these differences remain statistically indistinguishable from zero. In conclusion, the imagined epidemic development in Denmark does not affect participants' likelihood of accepting a vaccine independently.

\section{Average attribute effects}

The average attribute effects on the probability of accepting a vaccine are plotted in Fig. 1 (Table S.5 in the SM provides the supporting regression table). On the vaccine characteristics, the results show that vaccines that provide $70 \%$ protection are about 13 percentage points more likely to be accepted compared to vaccines with $50 \%$ protection, while vaccines with $90 \%$ protection are about 24 percentage points more likely to be accepted. We find similar but smaller effects for vaccines with lower risks of serious side-effects. Vaccines where 1 in 10,000 and 1 in 100,000 are hit by serious side-effects, are about 8 and 15 percentage points more likely to be accepted, respectively, compared to vaccines where the risk is 1 in 1,000 .

On the characteristics related to vaccine development, we observe that participants attach high importance to an increased testing period. Test periods of 6 and 12 months as compared to 3 months, increase vaccine acceptance by about 5 and 8 percentage points, respectively. We find a similar but smaller premium of about 3.5 and 7 percentage points, respectively, for vaccines that 100,000 and $1,000,000$ as compared to 10,000 have already received. Vaccines that are produced abroad face a penalty of about 2.5 percentage points (UK) and 6.5 percentage points (USA).

On the vaccination strategy characteristics, we find that compared to vaccines recommended by health authorities, a recommendation from your own doctor or the government face penalties of about 3 percentage points each, while vaccines recommended by a researcher are about 6 percentage points less likely to be accepted. Similarly, we observe some difference with respect to vaccination place with a penalty of about 3 and 3.5 percentage points, respectively, for getting the vaccination at the pharmacy and regional hospital compared to your own doctor. Moving to the two remaining factors, we find no difference heterogeneity in vaccine acceptance with respect to vaccination time or with respect to making appointments.

Taken together, the results demonstrate that vaccine acceptance indeed depends on characteristics related to the vaccine itself, its development, and the vaccination strategy. Specifically, preferences over vaccines are structured by seven main factors: vaccine efficacy, side-effects, number of previously vaccinated, testing period, production country, endorsements, and place of vaccination administration.

\section{Effect heterogeneity}


Do vaccine preferences vary across people with different psychological dispositions? As discussed above, we might expect that the attribute effects are driven by those who given their background characteristics are already more inclined to be vaccinated. For instance, we know from previous studies that those who feel personally at risk are more likely to accept vaccines $[5,7,8,9]$. This could suggest an interaction, where the attribute effects would be strongest for those who perceive a high degree of personal risk. In order to test for interactions between participants' psychological dispositions and the vaccine attribute effects, we split the main analysis according to four recognized psychological vaccination dispositions, including 1) institutional trust, 2) personal COVID-19 worry, 3) vaccine motivations, and 4) vaccine worry (see Table S.4 in the SM for details on the measurements of these psychological factors).

Figure 2 shows the results of these subgroup analyses (Table S.6 in the SM reports the supporting regression table). Overall, we observe a striking homogeneity in the attribute effects across the subgroups that are below the median (blue circles) and above the median (yellow triangles) on these psychological dispositions, respectively. Additional analyses using more fine-grained subgroups show that results are similarly homogenous (see Figure S.5-Figure S.8).

Do the vaccine attribute effects vary across demographic subgroups? In order to test for interactions between participant demographic and the effects of vaccine attributes, we stratify the main analyses by participants' sex, age, education, and geographical location (see Figure S.9 and Figure S.10 in the SM). Overall, we find that the attribute effects are broadly similar across different subgroups. This suggests that there is a consensus-among females and males, young and old, low and high educated, and across geographic regions-on which vaccines are preferred over others. However, there is one notable exception to this homogeneity. Hence, the age panel shows that the young drive the estimated impact of side-effects. This aligns with studies making the argument that SARS-COV-2 has an asymmetric age-profile [31], implying that the elderly would be more likely to accept any vaccine.

Although the epidemic context did not independently affect vaccine acceptance (see Figure S.2), the attribute effects might still vary across contexts. For example, vaccine safety might matter less in the context of an increasing epidemic and more in the context of a decreasing epidemic. However, we observe that the estimated effects remain fundamentally similar across contexts (see Figure S.11). Relatedly, we ask if the attribute effects vary across different vaccine profiles. Consistent with psychological research on decision-making under uncertainty, we might, for instance, expect that the influence of vaccine safety characteristics (e.g., side-effects) is largest when vaccine efficacy is relatively low. However, we find no substantively meaningful first-order interaction among any of the conjoint attributes. Altogether, the absence of such interaction effects suggest that participants consider the different characteristics independently from each other (see Figure S.12-Figure S.20).

\section{Predictions of acceptance}

To better understand the substantive meaning of these results, Fig. 3 illustrates the predicted probability of vaccine acceptance based on the model estimates from our baseline model in Fig. 1. In particular, we extract the probability of acceptance at the minimum, 25th percentile, median, 75th percentile, and the maximum. The acceptance rate of the least likely vaccine is about 23 percent (95\% Cl: $19-28)$, while the rate of the most likely vaccine is about 95 percent (95\% Cl: 90-99). The intermediate vaccines at the 25th percentile, the median, and the 75th percentile are predicted to be accepted by about 50 percent ( $95 \% \mathrm{Cl}: 46-55), 60$ percent (95\% Cl: 55-64), and 69 percent (95\% Cl: 65-74), respectively.

Mirroring the results from Fig. 1, we see a large jump in the expected acceptance from the minimum (about 23 percent acceptance) to the 25 th percentile (about 50 percent acceptance) driven by changes in the vaccine safety attributes. Moving from the minimum to the 25th percentile, we most notably see a decrease in side-effects, an increase in the number of previously vaccinated, and a change in production country from the US to the UK. Moving further up the distribution to the median (about 60 percent acceptance), we see that the jump in acceptance is driven by a change in vaccine efficacy from 50 to 90 percent. Comparing the 75th percentile (about 69 percent acceptance) to the median, we see an increase in vaccine acceptance of about 9 percentage points driven by increases in vaccine safety in terms of a lower risk of serious side-effects and an increased test period. Finally, we see that the increase from the 75th percentile to the maximum (about 95 percent acceptance) is driven by: 1) an increased test period, 2) an increasing number of vaccinated, 3) that the vaccine is produced in Denmark, 3) that the authorities recommend the vaccine, 4) that vaccination is at your own doctor, 5) that you do not have to book an appointment.

Page 6/12 


\section{Discussion}

These findings have key implications for our understanding of COVID-19 vaccine acceptance and, specifically, the results establish a strong hierarchy between different factors related to vaccines. Most important are the factors that make the use of a specific vaccine worthwhile from a cost-benefit perspective. We thus find strong evidence that a vaccine's expected protection and safety are crucial determinants of vaccine acceptance. Factors relating to the development of a vaccine have a smaller effect, suggesting that such factors can contribute to confidence in the vaccines beyond there mere fact that they have been approved by the national health authorities. The factors relating to the implementation of the vaccination strategy matters the least for acceptance. Still, there are some effects and, for health authorities, these effects may be particularly important to note as the implementation is fully under their control. As compared to endorsements by the health authorities, we observe a penalty from endorsements of e.g., researchers and the government. We similarly see that place of vaccine administration matters. That is, being able to be vaccinated at your own doctor is attached with a premium compared to more centralized alternatives.

Importantly, we observe a striking consensus across different subsets of the population about which vaccines are preferred over others. This is crucial. Thus, it shows that the potential positive effects of these characteristics are not limited to the subgroups in the population that are most enthusiastic about vaccines given their background characteristics. Instead, the estimated effects were strikingly homogenous across groups. This reveals that these characteristics can have positive effects also among those groups who are viewed to be more difficult to convince to be vaccinated.

In terms of study limitations, it is important to note that while our experimental design (i.e., the randomization of attributes) ensures the study's internal validity, there are potentially issues in relation to external validity. However, five factors help alleviate concerns over the generalizability of our study beyond the sample. First, we are aware of one external validation test that shows that the single-conjoint we applied can achieve high external validity in replicating real-world behavior and reducing social desirability bias [30]. Second, the number of profiles that each respondent accepted in their conjoint tasks is highly correlated with respondents' general vaccine attitudes as measured by the vaccine motivation and vaccine worry scales. Thus, a one standard deviation increase in vaccine trust is associated with about a 13.5 percentage points increase in the likelihood of vaccine acceptance while a standard deviation increase in vaccine worry is associated with a decrease of about 15 percentage points. Overall, this suggest that respondents' judgments of individual vaccines are closely linked to their broader psychological dispositions. Third, as shown above the effects are strikingly homogenous across subgroups of respondents, implying that it is unlikely that our results would have been substantially different had our sample contained different distributions on age, gender, education, institutional trust, geography, and psychological motivations. Moreover, our results remain fundamentally similar if we do not use weights to post-stratify our data for analysis (see Figure S.4). Fourth, it is important to note, that the majority of respondents did not either categorically reject or accept all of their assigned vaccine profiles.[1] This suggests that our findings on how citizens condition their acceptance on the basis of specific vaccine attributes is not only applicable on the margins but have broadly meaningful implications for public acceptance of vaccines and the end-goal of herd immunity. Finally, given the robustness of these design-specific concerns, it is crucial to note the very high quality of our sample. Thus, the random sampling from the central database of social security numbers combined with a relatively high response rate of about 39 percent ensure that results should be representative for the adult Danish population.

\section{Footnote:}

[1] Thus, 52 percent of our respondents accepted between 1-3 of their vaccine offers while about 14 percent categorically rejected all vaccines and about 34 percent accepted all.

\section{Conclusions}

Overall, the findings reveal both opportunities and challenges for policy-makers who are struggling to develop strategies and communicate in ways that ensure high vaccine uptake in the population. The public's strong preference for vaccines with very high efficacy points to a mounting challenge for ensuring high vaccine uptake. Yet, the results also provide evidence that there might be a pathway to naturally alleviate this challenge. Thus, as more people are vaccinated and the "testing period" of the vaccines are naturally extended, it simultaneously implies an increase in vaccine safety. Our results suggest that this in itself will 
increase acceptance (in particular, if the roll-out only reveals marginal side-effects). Consistent with this, observational research show that acceptance of vaccines against SARS-Cov-2 has increased in multiple countries from September 2020 to February 2021 as vaccines were rolled out (Lindholt et al., 2021). Furthermore, the results indicate that vaccine acceptance can be increased if politicians leave it to the health authorities to communicate about recommendations. Hence, vaccines recommended by the government, a doctor, or a researcher faced clear penalties as compared to vaccines promoted by the health authorities. Importantly, the present results suggest that such factors will increase acceptance, even among those who otherwise are predisposed to be hesitant.

\section{Declarations}

Ethics approval and consent: The study complies with Aarhus University's Code of Conduct as well as the Committee Act of the Danish National Committee of Health Research Ethics, which states that 'Surveys using questionnaires and interviews that do not involve human biological material (section 14(2) of the Committee Act)' are exempted from ethics approval (https://en.nvk.dk/how-to-notify/what-to-notify).

All procedures performed in this study involving human participants were performed in accordance with the ethical standards of The Danish Code of Conduct for Research Integrity.

Consent for publication: Not applicable.

Availability of data and materials: Data and code to reproduce all results are available at Open Science Framework: https://osf.io/8jz7r/.

Competing Interest: The authors declare that they have no known competing financial interests or personal relationships that could have appeared to influence the work reported in this paper.

Funding: This data collection was funded the Danish Health Authorities (Sundhedsstyrelsen) and researchers' salaries was funded by grant CF20-0044 from the Carlsberg Foundation to Michael Bang Petersen.

Author contributions: F.J.J., A.B., and M.B.P conceived of the research idea; F.J.J. analyzed the data; F.J.J., A.B., and M.B.P interpreted the results; F.J.J. drafted the article while A.B. and M.B.P. provided critical revisions of the intellectual content.

Acknowledgements: Thorkil Klint, Tomas Bargisen, and Christian Fischer Vestergaard (from the Survey firm Epinion) provided critical contributions to the study design and data collection and provided helpful comments on analyses.

\section{References}

1. WHO. 2020. WHO Director-General's opening remarks at the media briefing on COVID-19 - 21 August 2020. https://www.who.int/dg/speeches/detail/who-director-general-s-openingremarks-at-the-media-briefing-on-covid-19--21august-2020

2. Britton, T., Ball, F., \& Trapman, P. (2020). A mathematical model reveals the influence of population heterogeneity on herd immunity to SARS-CoV-2. Science, 369, 846-849.

3. Callaghan, Timothy and Moghtaderi, Ali and Lueck, Jennifer A. and Hotez, Peter J. and Strych, Ulrich and Dor, Avi and Franklin Fowler, Erika and Motta, Matt. 2020. Correlates and Disparities of COVID-19 Vaccine Hesitancy. SSRN: https://ssrn.com/abstract=3667971 or http://dx.doi.org/10.2139/ssrn.3667971

4. Lazarus, J. V., Ratzan, S. C., Palayew, A., Gostin, L. O., Larson, H. J., Rabin, K., \& El Mohandes, A. (2020). A global survey of potential acceptance of a COVID-19 vaccine. Nature medicine, 1-4.

5. Lindholt, M. F., Jørgensen, F., Bor, A., \& Petersen, M. B. (2021). Public acceptance of COVID-19 vaccines: cross-national evidence on levels and individual-level predictors using observational data. BMJ open, 11(6), e048172.

6. Roozenbeek, J., Schneider, C. R., Dryhurst, S., Kerr, J., Freeman, A. L., Recchia, G., ... \& Van Der Linden, S. (2020). Susceptibility to misinformation about COVID-19 around the world. Royal Society open science, 7(10), 201199. 
7. Dror, A. A., Eisenbach, N., Taiber, S., Morozov, N. G., Mizrachi, M., Zigron, A., \& Sela, E. (2020). Vaccine hesitancy: the next challenge in the fight against COVID-19. European journal of epidemiology, 35(8), 775-779.

8. Wong, L. P., Alias, H., Wong, P. F., Lee, H. Y., \& AbuBakar, S. (2020). The use of the health belief model to assess predictors of intent to receive the COVID-19 vaccine and willingness to pay. Human vaccines \& immunotherapeutics, 1-11.

9. Hacquin, A. S., Altay, S., de Araujo, E., Chevallier, C., \& Mercier, H. (2020). Sharp rise in vaccine hesitancy in a large and representative sample of the French population: reasons for vaccine hesitancy.

10. Johnson, T., Dawes, C., Fowler, J., \& Smirnov, O. (2020). Slowing COVID-19 transmission as a social dilemma: Lessons for government officials from interdisciplinary research on cooperation. Journal of Behavioral Public Administration, 3(1).

11. Pfattheicher, S., Nockur, L., Böhm, R., Sassenrath, C., \& Petersen, M. B. (2020). The emotional path to action: Empathy promotes physical distancing and wearing of face masks during the COVID-19 pandemic. Psychological Science, 31(11), 1363-1373.

12. Tomljenovic, H., Bubic, A., \& Erceg, N. (2020). It just doesn't feel right-the relevance of emotions and intuition for parental vaccine conspiracy beliefs and vaccination uptake. Psychology \& Health, 35(5), 538-554.

13. Freeman, D., Waite, F., Rosebrock, L., Petit, A., Causier, C., East, A., .. \& Bold, E. (2020). Coronavirus conspiracy beliefs, mistrust, and compliance with government guidelines in England. Psychological Medicine, 1-30.

14. Michie, S., West, R., \& Harvey, N. (2020). The concept of "fatigue" in tackling covid-19. bmj, 371.

15. WHO. 2014. Report of the SAGE Working Group on Vaccine Hesitancy.

http://www.who.int/immunization/sage/meetings/2014/october/SAGE_working_group_revised_report_vaccine_hesitancy.pdf

16. Betsch, C., Schmid, P., Heinemeier, D., Korn, L., Holtmann, C., \& Böhm, R. (2018). Beyond confidence: Development of a measure assessing the $5 \mathrm{C}$ psychological antecedents of vaccination. PloS one, 13(12), e0208601.

17. Determann, D., Korfage, I. J., Lambooij, M. S., Bliemer, M., Richardus, J. H., Steyerberg, E. W., \& de Bekker-Grob, E. W. (2014). Acceptance of vaccinations in pandemic outbreaks: a discrete choice experiment. PloS one, 9(7), e102505.

18. Kaplan, R. M., \& Milstein, A. (2021). Influence of a COVID-19 vaccine's effectiveness and safety profile on vaccination acceptance. Proceedings of the National Academy of Sciences, 118(10).

19. Schwarzinger, M., Watson, V., Arwidson, P., Alla, F., \& Luchini, S. (2021). COVID-19 vaccine hesitancy in a representative working-age population in France: a survey experiment based on vaccine characteristics. The Lancet Public Health.

20. Kreps, S., Prasad, S., Brownstein, J. S., Hswen, Y., Garibaldi, B. T., Zhang, B., \& Kriner, D. L. (2020). Factors associated with US adults' likelihood of accepting COVID-19 vaccination. JAMA network open, 3(10), e2025594-e2025594.

21. Motta, M. (2021). Can a COVID-19 vaccine live up to Americans' expectations? A conjoint analysis of how vaccine characteristics influence vaccination intentions. Social Science \& Medicine, 113642.

22. Guo N, Zhang G, Zhu D, Wang J, Shi L. The effects of convenience and quality on the demand for vaccination: results from a discrete choice experiment. Vaccine. 2017;35(21):2848-2854.

23. Veldwijk J, Lambooij MS, Bruijning-Verhagen PCJ, Smit HA, de Wit GA. Parental preferences for rotavirus vaccination in young children: a discrete choice experiment. Vaccine. 2014;32(47):6277-6283.

24. de Bekker-Grob EW, Hofman R, Donkers B, et al. Girls' preferences for HPV vaccination: a discrete choice experiment. Vaccine. 2010;28(41):6692-6697.

25. de Bekker-Grob EW, Veldwijk J, Jonker $M$, et al. The impact of vaccination and patient characteristics on influenza vaccination uptake of elderly people: a discrete choice experiment. Vaccine. 2018;36(11):1467-1476

26. WHO. 2021. Draft landscape of COVID-19 candidate vaccines. https://www.who.int/publications/m/item/draft-landscape-ofcovid-19-candidate-vaccines Accessed 16 ${ }^{\text {th }}$ March 2021.

27. Bansak, K., Hainmueller, J., Hopkins, D. J., \& Yamamoto, T. (2018). The number of choice tasks and survey satisficing in conjoint experiments. Political Analysis, 26(1), 112-119.

28. Bansak, K., Hainmueller, J., \& Hangartner, D. (2016). How economic, humanitarian, and religious concerns shape European attitudes toward asylum seekers. Science, 354(6309), 217-222.

29. Hainmueller, J., \& Hopkins, D. J. (2014). Public attitudes toward immigration. Annual review of political science, 17, 225-249. 
30. Hainmueller, J., Hangartner, D., \& Yamamoto, T. (2015). Validating vignette and conjoint survey experiments against real-world behavior. Proceedings of the National Academy of Sciences, 112(8), 2395-2400.

31. Jørgensen, F., Bor, A., \& Petersen, M. B. (2021). Compliance without fear: Individual-level protective behaviour during the first wave of the COVID-19 pandemic. British Journal of Health Psychology, 26(2), 679-696.

\section{Figures}

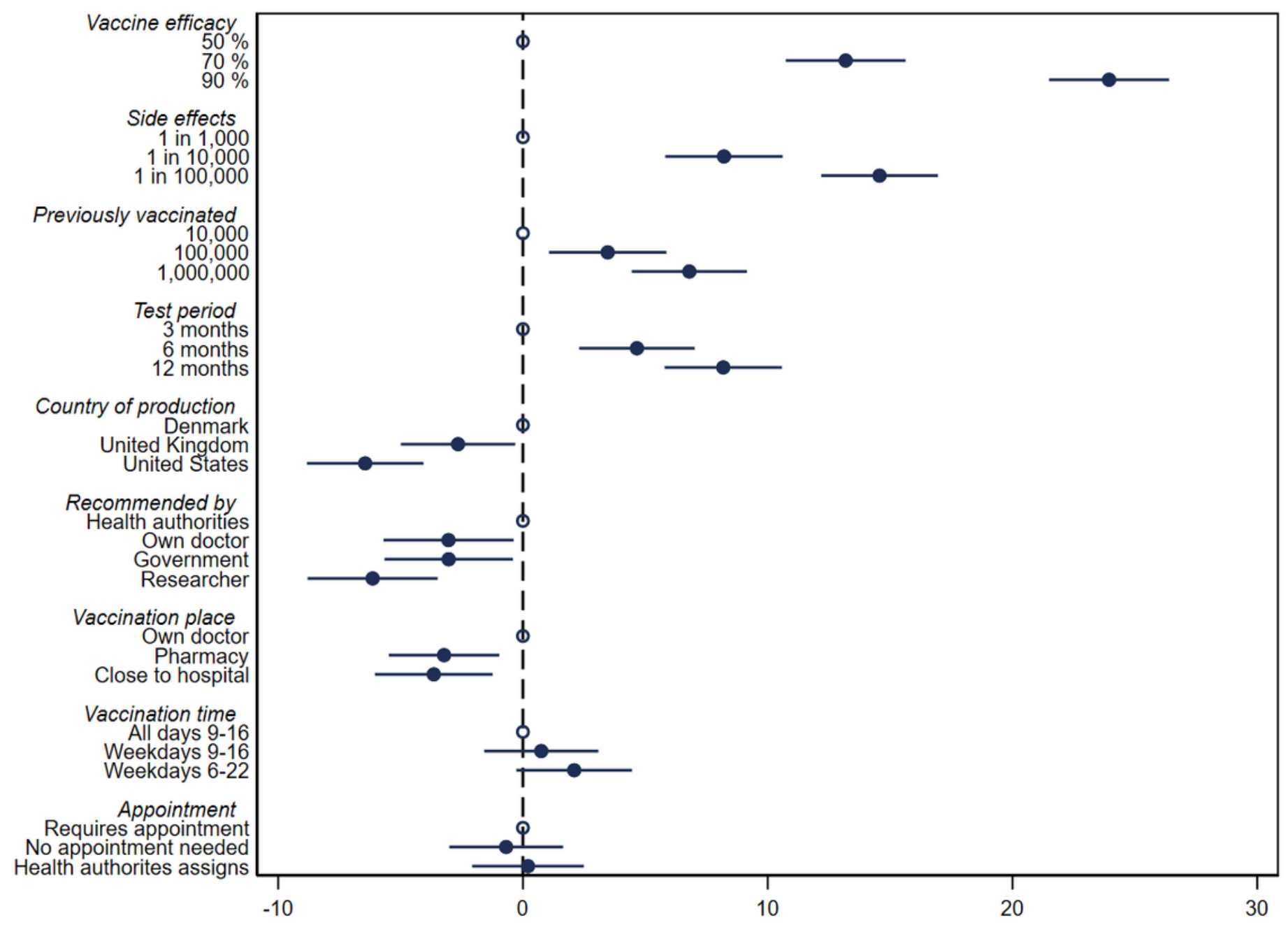

Figure 1

Effects of vaccine attributes on the probability of vaccine acceptance

Note: filled circles with horizontal lines indicate point estimates with cluster-robust $95 \% \mathrm{Cl}$ from linear (weighted) least square regression. Hollow circles on the dashed zero line denote the reference category for each vaccine attribute. 

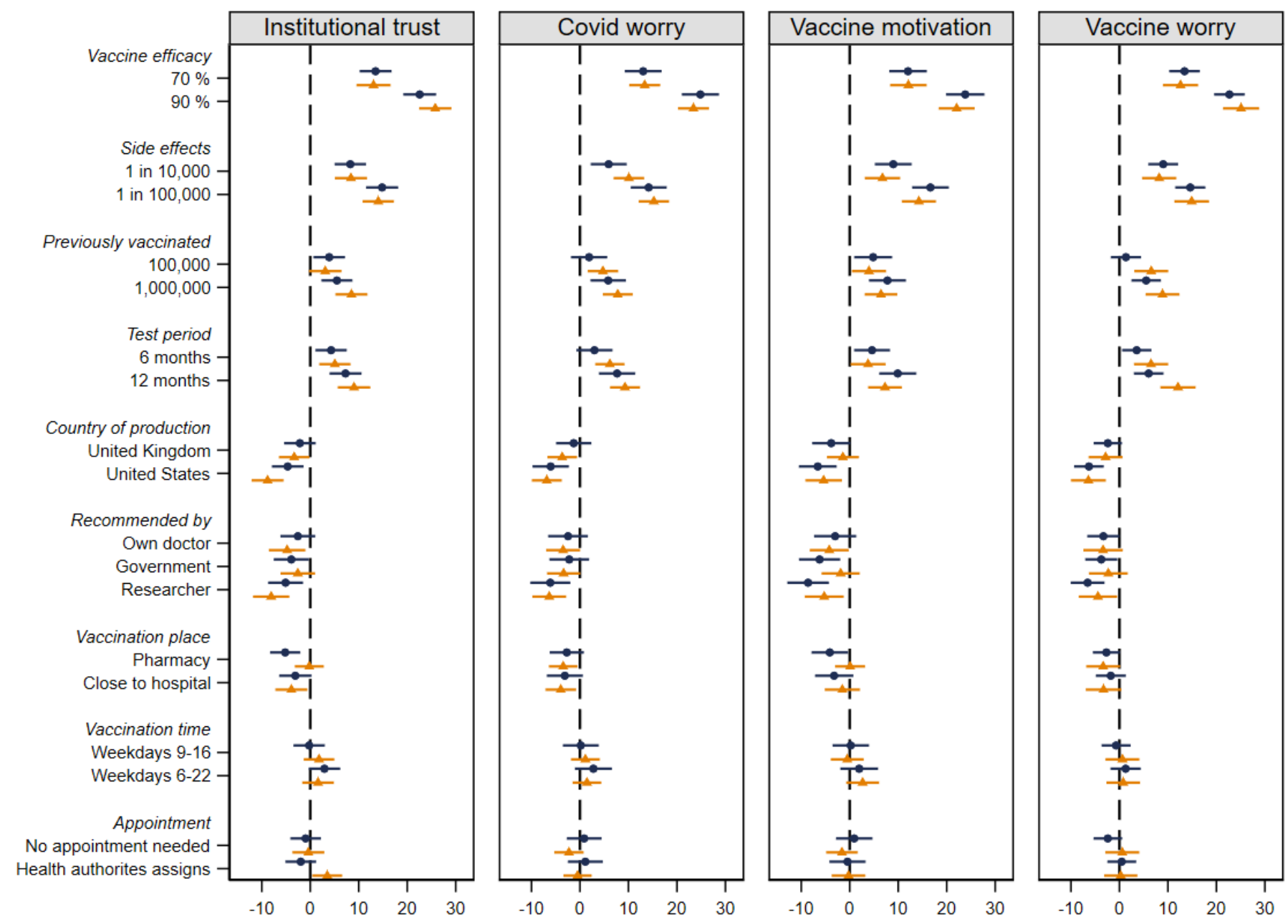

\section{Figure 2}

Vaccine attribute effects on the probability of vaccine acceptance across subgroups

Note: blue circles represent attribute effect estimates for participants below the median on each psychological disposition, while yellow triangles represent attribute effect estimates for participants above the median. Horizontal lines are cluster-robust $95 \% \mathrm{Cl}$. Linear (weighted) least square regression. 


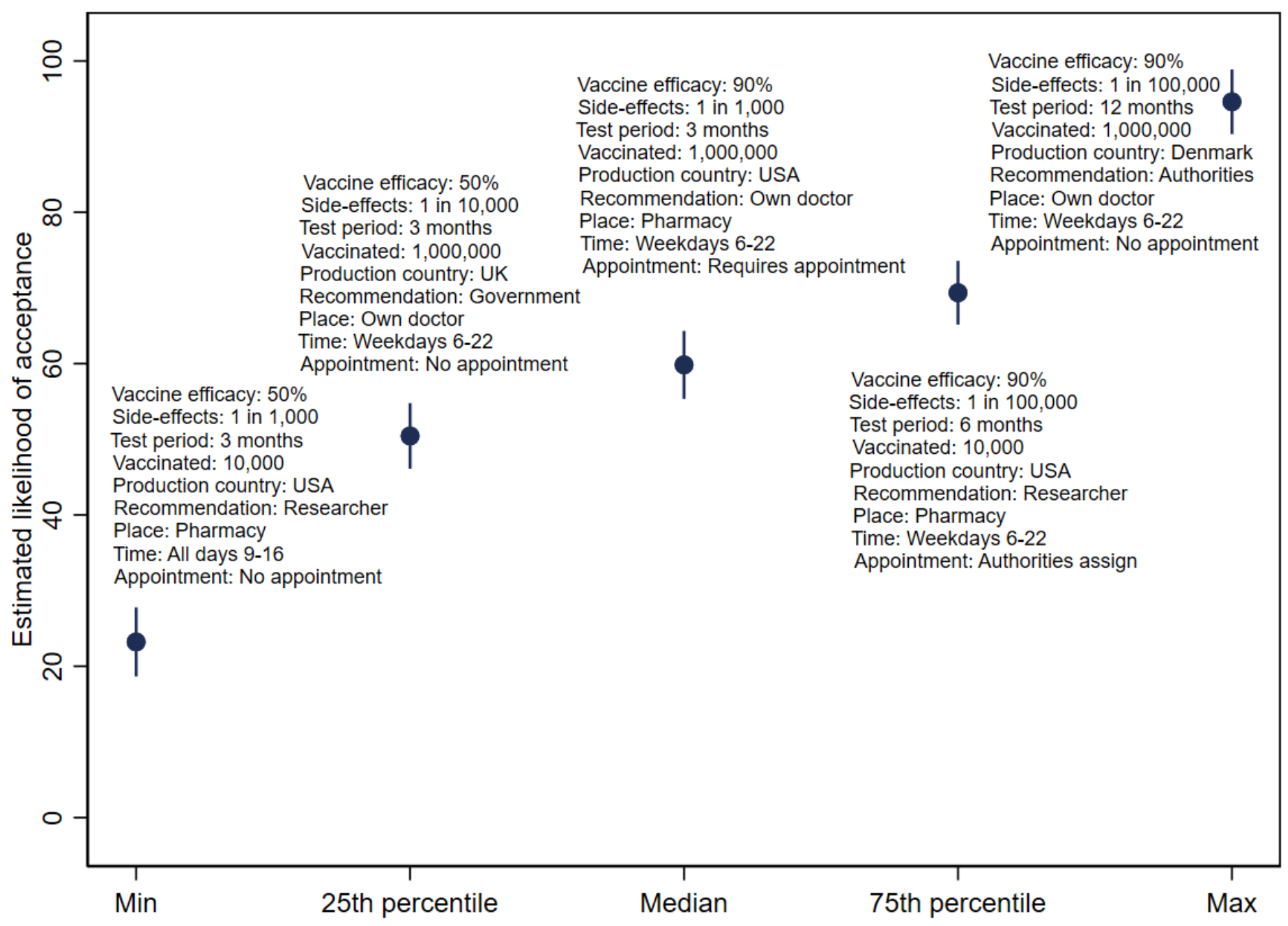

Figure 3

Estimated probability of vaccine acceptance for selected vaccine profiles

Note: filled circles with horizontal lines indicate estimated point prediction with cluster-robust $95 \% \mathrm{Cl}$ from linear (weighted) least square regression.

\section{Supplementary Files}

This is a list of supplementary files associated with this preprint. Click to download.

- Supplementarymaterial.docx 